\title{
Investigation of Deprotonation Reactions on Globular and Denatured Proteins at Atmospheric Pressure by ESSI-MS
}

\author{
David Touboul, Matthias Conradin Jecklin, and Renato Zenobi \\ Department of Chemistry and Applied Biosciences, ETH Zürich, Zürich, Switzerland
}

Deprotonation reactions of multiply charged protein ions have been studied by introducing volatile reference bases at atmospheric pressure between an electrosonic spray ionization (ESSI) source and the inlet of a mass spectrometer. Apparent gas-phase basicities $\left(\mathrm{GB}_{\mathrm{app}}\right)$ of different charge states of protein ions were determined by a bracketing approach. The results obtained depend on the conformation of the protein ions in the gas phase, which is linked to the type of buffer used (denaturing or nondenaturing). In nondenaturing buffer, the $\mathrm{GB}_{\mathrm{app}}$ values are consistent with values predicted by the group of Kebarle using an electrostatic model (J. Mass Spectrom. 2002, 38, 618) based on the crystal structures, but taking into account salt bridges between ionized basic and acidic sites on the protein surface. A new basicity order for the most basic sites was therefore obtained. An excellent agreement with the charge residue model (CRM) is obtained when comparing the observed and calculated maximum charge state. Decharging of the proteins in the electrosonic spray process could be also useful in the study on noncovalent complexes, by decreasing repulsive electrostatic interactions. A unified mechanism of the ESSI process is proposed. (J Am Soc Mass Spectrom 2008, 19, 455-466) (ㅇ 2008 American Society for Mass Spectrometry

$\mathrm{E}$ lectrospray ionization mass spectrometry (ESIMS) [1] rapidly became established as the method of choice for the production of large biomolecular ions in the gas phase. For proteins, ESI leads to the formation of multiply charged ions, both in the positive and negative ion modes. The charge-state distribution is directly related to the conformation of the macromolecular ions [2] and the proton transfer reactions in the gas phase [3]. Thermodynamic information, such as values for the gas-phase acidity/basicity (GA/GB), is essential to the understanding of the proton transfer processes and generation of ions by ESI in general. The intrinsic gas-phase basicity of a $[\mathrm{M}+(n-1) \mathrm{H}]^{(n-1)+}$ ion or the gas-phase acidity of a $[\mathrm{M}+\mathrm{nH}]^{n+}$ ion can be defined as the free enthalpy change $\Delta G^{0}$ of the reaction:

$$
[M+n H]^{n+} \rightarrow[M+(n-1) H]^{(n-1)+}+H^{+}
$$

Since this reaction proceeds with a reverse activation barrier, due to Coulombic repulsion between the two charges product ions, the terms "apparent gas-phase acidity" $\left(\mathrm{GA}_{\mathrm{app}}\right)$ and "apparent gas-phase basicity" $\left(\mathrm{GB}_{\mathrm{app}}\right)$ are more appropriate. The $\mathrm{GA}_{\mathrm{app}}$ is defined as the sum of the $\Delta \mathrm{G}$ and the energy of the activation barrier, considered as a negative term [4].

Address reprint requests to Professor Renato Zenobi, Department of Chemistry and Applied Biosciences, ETH Zurich, HCI E 329, CH-8093 Zürich, Switzerland. E-mail: ZENOBI@org.chem.ethz.ch
Determination of $\mathrm{GB}_{\mathrm{app}} \mathrm{s}$ of peptides and proteins provides important information which is analogous to measurements of $\mathrm{pKa}$ in solution. For example, pKa values are greatly affected by intramolecular interactions, and the conversion of the denatured to the native form maximizes these interactions. Similarly, $\mathrm{GB}_{\mathrm{app}}$ values will also reflect the conformation of the ions in the gas phase.

For more than 10 years, research groups have tried to determine apparent gas-phase basicities of peptides and proteins. For small peptides, like bradykinin and its des-arginine analogues, the kinetic method can be directly used to determine $\mathrm{GB}_{\text {app }}$ values $[5,6]$. The energy of the activation barrier was obtained by measuring the kinetic energy release to calculate the intrinsic GB of two different charge states. Unfortunately, this method is quite difficult to transfer to multiply charged protein ions.

A bracketing technique, consisting of evaluating the qualitative or quantitative rate of proton transfer between a neutral volatile base and protein ions was introduced to measure $\mathrm{GB}_{\text {app }}$ values of multiply charges ions [4, 7, 8]. In terms of instrumentation, different systems can be used to observe the deprotonation reactions. High-pressure mass spectrometry (HPMS) experiments were designed by the group of Kebarle to study cluster formation from small organic or inorganic ions, and water in particular $[9,10]$. This apparatus was then modified by the group of Smith 
[11, 12], who developed a new Y shaped capillary interface/ flow reactor connected to a quadrupole mass spectrometer. One inlet was fitted with an ESI source while the second inlet was used to introduce the basic reactant (volatile amines or water vapor) at a known flow rate. Due to the poor ionization efficiency with nondenaturing buffer and low ion transmission of the quadrupole in the high mass range, mostly qualitative data were obtained for denatured proteins. A few years later, the groups of Cassady [13-15] and Williams [2,4] used ESI-FT-ICR mass spectrometers to precisely determine the $\mathrm{GB}_{\mathrm{app}} \mathrm{s}$ of multiply charged proteins by the bracketing method. A defined protein charge state is preselected and allowed to react with volatile base in the ICR cell. The kinetics of the deprotonation is then studied by recording the signal intensities of different charge states of a protein with different volatile bases. The rate constant of the deprotonation reaction was found to increase with increasing charge state of the ion and with increasing basicity of the amine [13]. Nevertheless, most of the experimental data dealt only with proteins sprayed from denaturing solution. With this technique, the ions must be stored during a long time (several tens of seconds), leading to a lack of sensitivity, and to difficulties to measure and control pressure and temperature in the cell. Moreover, Gross et al. showed that at least two distinct $\mathrm{GB}_{\text {app }}$ values could be attributed to charge states 7 through 11 of disulfide-intact lysozyme sprayed from nondenaturing buffer, due to different conformations of the gas-phase ions that were stored for a long time in this experiment [2]. Fast folding/unfolding equilibria in the gas phase were recently described when protein ions are stored in an ion trap [16] or an ICR cell [17] leading to the coexistence of different ion populations.

Efforts have also been made to develop models for predicting the $\mathrm{GB}_{\text {app }}$ values of globular protein ions. In 1995, the group of Williams described for the first time a simple electrostatic model capable of calculating the apparent and intrinsic GB for each charge state of a protein $[4,18]$. However, recent work by Gronert, based on ab initio calculations, showed that the equation for the two-proton system used by Williams and coworkers predicts $\mathrm{GB}_{\text {app }}$ values that are too low [19-21]. In 2002, Kebarle and coworkers developed a new electrostatic model giving access to calculated $\mathrm{GB}_{\mathrm{app}}$ of folded proteins [22]. This calculation is based on a correction of the intrinsic GB of each basic site (arginine, lysine and histidine) by taking into account the Coulombic repulsion energy between two positively charged sites, the charge/(permanent or induced) dipole interaction energy and some entropic terms. All of these data were obtained using crystal structures of the proteins, and gave an estimated error of $\pm 5 \mathrm{kcal} \cdot \mathrm{mol}^{-1}$.

As alternative to the delicate FT-ICR measurements, the reference base can be introduced directly into the solution. The first observations based on this idea were made by the group of Laprévote in 2001 [23]. They showed that triethylammonium bicarbonate (TEAB) buffer leads to a reduction of the charge state of the proteins and to a stabilization of noncovalent protein complexes. This idea was taken up, extended, and rationalized by Verkerk et al. in 2003 to demonstrate the validity of their model on three different proteins [24]. Encouraging results were only obtained for lysozyme and ubiquitin, while the agreement was poor for cytochrome $c$ where a too high-temperature on the capillary led to denaturation. Recently, Heck and coworkers used "proton sponges" to neutralize the most basic residues on the proteins [25]. The results obtained are consistent with former observations. This data were interpreted in terms of $\mathrm{GB}_{\mathrm{app}}$. Nevertheless, all of these experiments were made using different inorganic or organic salts in the solvent, which made it impossible to determine the relative influence of solution and gas-phase reactions, especially in the last stages of the droplet fission and evaporation process. Therefore, a new experimental set-up had to be found to separate all of these effects and confirm or discount the model developed by Kebarle and coworkers.

Electrosonic spray ionization (ESSI) was developed by Cooks and coworkers in 2004 [26]. The principle is to use a standard micro ESI source with a supersonic nebulizing gas. Narrower charge-state distributions and peak widths were shown in comparison with traditional ESI. Evidence was provided that fully desolvated ions can be generated at atmospheric pressure before they enter in the mass spectrometer.

Very recently, our group has introduced a fast and sensitive mass spectrometric measurement of the $\mathrm{GB}_{\text {app }}$ of peptides and proteins based on ESSI-MS [27]. Our set-up is quite simple similar to the Y shaped capillary interface/flow reactor developed by the group of Smith in the early 1990s: vapor of reference bases (volatile amines and alcohols) are allowed to react simultaneously with all the charge states of the protein generated by ESSI at atmospheric pressure, before them being sampled into the mass spectrometer. Due to a high reference base pressure, close to the saturation pressure, and a sufficient reaction time, estimated to few hundreds of $\mu \mathrm{s}$, a high collision rate is ensured. One might ask whether or not a thermodynamically allowed reaction goes to completion using this arrangement, because is not possible to control the partial pressure of the volatile bases. This is definitely the case: when the distance between the spray tip and the entrance of the mass spectrometer was increased and numerous tubes containing the volatile base were placed all along the spray axis, no change in the extent of the deprotonation reaction was observed for every base investigated, i.e., there is a sufficient number of reactive collisions between the neutral base molecules and the ions before they are sampled into the mass spectrometer. In that case, only a 30-60 s acquisition is necessary to acquire a mass spectrum for verifying the presence or absence of a proton transfer to the reference base. This technique was validated by comparing the $\mathrm{GB}_{\text {app }}$ measured by ESSI with those obtained by the 
deprotonation reaction method or the kinetic method. Our experimental set-up based on ESSI-MS allows us to determine $\mathrm{GB}_{\mathrm{app}}$ of peptides and proteins at atmospheric pressure with good sensitivity (for concentrations less than $10 \mu \mathrm{M}$ in denaturing or nondenaturing buffer), very good precision (less than $2 \%$; the deviation is calculated for deprotonation rates of $10 \%$ and $90 \%$.) and in a short time (less than $30 \mathrm{~min}$ to screen up to 23 volatile bases).

Here we present gas-phase deprotonation reactions and $\mathrm{GB}_{\mathrm{app}}$ measurements at atmospheric pressure by ESSI-MS for seven different model proteins (ubiquitin, lysozyme, ribonuclease A, $\alpha$-lactalbumin, cytochrome $c$, human carbonic anhydrase II, creatine kinase) in native and/or denaturing buffer. Our experimental $\mathrm{GB}_{\mathrm{app}}$ values are in good agreement with predicted values, taking into account some corrections due to salt bridges on the protein surface. Our experiments give evidence of the validity of the Rayleigh limiting charge theory, also known as the Charge Residue Model (CRM) [28, 29]. A unified model of the electrosonic spray process for globular proteins can be proposed and extended to normal electrospray.

\section{Experimental}

\section{Materials and Sample Preparation}

Ubiquitin from bovine erythrocytes, lysozyme from hen egg white, ribonuclease A from bovine pancreas, $\alpha$ lactalbumin from bovine milk, cytochrome $c$ from equine heart, human carbonic anhydrase II, creatine phosphokinase I from rabbit muscle, human hemoglobin, and all of the volatile bases used (methanol, ethanol, 2-propanol, furan, benzonitrile, THF, anisole, ammonia, methylamine, allylamine, ethylamine, propylamine, butylamine, isopropylamine, isobutylamine, hexylamine, pyridine, 3-methylpyridine, 4-methylpyridine, cyclohexylamine, diethylamine, piperidine, di-isopropylamine, triethylamine, tripropylamine, tributylamine) were purchased from Sigma (Sigma-Aldrich Chemie $\mathrm{GmbH}$, Buchs, Switzerland).

Ten $\mu \mathrm{M}$ protein solutions were desalted using $6 \mathrm{kDa}$ cutoff ultrafiltration cartridges (Micro Bio-Spin 6; BioRad, Hercules, CA) equilibrated with a $10 \mathrm{mM}$ ammonium bicarbonate buffer at $\mathrm{pH} 7.2$ for experiments in nondenaturing buffer. A water/methanol/acetic acid (50/50/1, vol/vol/vol) mixture was used for experiments in denaturing buffer. For the disulfide reduced lysozyme sample, lysozyme was dissolved and purified in $10 \mathrm{mM}$ ammonium bicarbonate. Then the disulfide bounds were reduced by adding dithiothreitol (final concentration $20 \mathrm{mM}$ ) and by boiling the solution during $30 \mathrm{~min}$ immediately before the MS analysis.

\section{Mass Spectrometry}

Experiments were performed on a hybrid quadrupole time-of-flight mass spectrometer (Q-TOf Ultima; Waters/
Micromass Ltd., Manchester, UK) equipped with a Zspray source. They were carried out at a source temperature of $50^{\circ} \mathrm{C}$, a cone voltage of $45 \mathrm{~V}$ and a RF1 lens voltage optimized to obtain the best mass resolution. Argon was used as collision gas and the collision energy parameter was typically set to $15-20 \mathrm{~V}$. The mass scale up to $6000 \mathrm{~m} / \mathrm{z}$ was calibrated by injecting a cesium iodide solution at $2 \mathrm{~g} \cdot \mathrm{L}^{-1}$ in water/2-propanol (1/1, vol/vol).

The ESSI source was home built [30] and close to the one first described by Takáts et al. [26]. The samples were delivered into the ESSI source by means of a syringe pump (Harvard 22; Harvard Apparatus $\mathrm{GmbH}$, March-Hugstetten, Germany) at a flow rate of 2-5 $\mu \mathrm{L} \cdot \mathrm{min}^{-1}$. The pressure of the nebulizing gas was set to 15-20 bar and the spray voltage was constant at $2 \mathrm{kV}$. The distance between the ion source and the entrance of the mass spectrometer was $\sim 10 \mathrm{~cm}$.

Eppendorf tubes, $1.5 \mathrm{~mL}$, containing the reference bases were placed between the ESSI source and the inlet of the mass spectrometer, slightly below the spray path of the ions. Like this, vapor of the volatile bases was introduced to react with the protein ions in the atmospheric pressure region. Protective masks can be used to avoid toxic gas exposure, even if a very small amount of base is used. Representative mass spectra were acquired during 30 to $60 \mathrm{~s}$, to obtain a good signal-tonoise ratio. The values of GB of the reference volatile bases are given in reference [27].

The $\mathrm{GB}_{\text {app }}$ of an individual charge state $n$ can be measured by monitoring the intensity ratio between the two successive charge states $[\mathrm{M}+\mathrm{nH}]^{n+}$ and $[\mathrm{M}+$ $(n-1) \mathrm{H}]^{(n-1)+}$. Criteria for the precise $\mathrm{GB}_{\text {app }}$ assignment will be discussed later in the text. The average charge state $(A C S)$ is given by: ACS $=\Sigma\left(N_{i} \times I_{i}\right) / \Sigma I_{i}$, where $N_{i}$ is the number of charges on each peak representing the protein, and $I_{i}$ is the absolute integral of each peak.

\section{Results and Discussion}

\section{Lysozyme, Ribonuclease $A$, and $\alpha$-Lactalbumin}

Figure 1 shows the mass spectra obtained with ESSI for disulfide-intact lysozyme (MW = 14,313 Da), in nondenaturing (Figure 1a) and denaturing buffer (Figure 1b), reacting with different volatile reference bases. The GBs of the bases are 195.7 (ammonia), 210.0 (ethylamine), 219.7 (diethylamine), and 227.3 (triethylamine) $\mathrm{kcal} \cdot \mathrm{mol}^{-1}$, according to the NIST database [31]. The first thing to notice is the successive disappearance of higher charge states and a gradual decrease of the average charge state (ACS) of the protein ions (Figure 2) with increasing GB of the reference base. In nondenaturing buffer, the charge state 7 is the highest peak in the spectra when using no base or ammonia. The main charge state is reduced to 6 with ethylamine and diethylamine and to 5 with triethylamine. The spectra with ethylamine and diethylamine are characterized by the presence of the charge 7 or 5 , respectively. The ion 

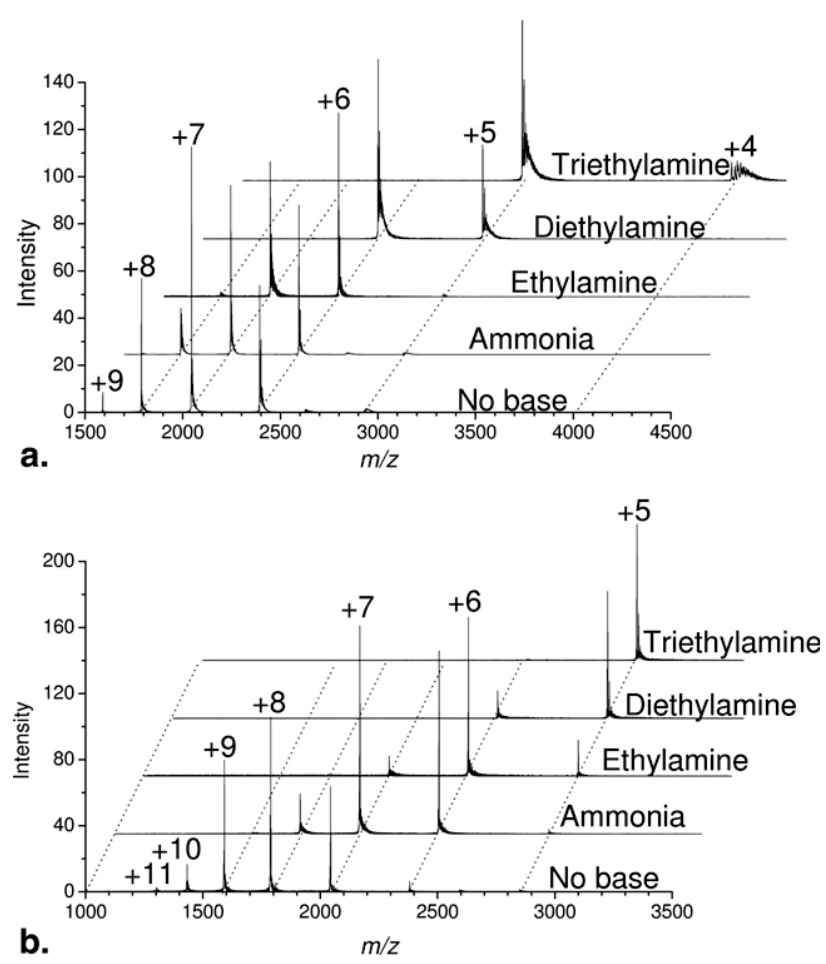

Figure 1. Representative mass spectra obtained by reacting lysozyme ions sprayed by ESSI from a nondenaturing (a) or a denaturing (b) buffer and different neutral volatile reference bases.

peak corresponding to $[\mathrm{M}+9 \mathrm{H}]^{9+}$ disappeared using ammonia as volatile reactive base, meaning that the $\mathrm{GB}_{\text {app }}$ of charge state 9 is below to the GB of ammonia. The $\mathrm{GB}_{\text {app }}$ of the charge states $n 8$ to 6 , of the $[\mathrm{M}+(n-$ $1)]^{(n-1)+}$ ion, respectively, can be estimated by monitoring the successive disappearance of the $[\mathrm{M}+\mathrm{nH}]^{n+}$ ion (Figure 3). The lines used in Figure 3 are drawn to guide the eye; the $\mathrm{GB}_{\mathrm{app}} \mathrm{s}$ were determined for a reduction of the $[\mathrm{M}+\mathrm{nH}]^{n+}$ ion intensity of $50 \%$. Normally,

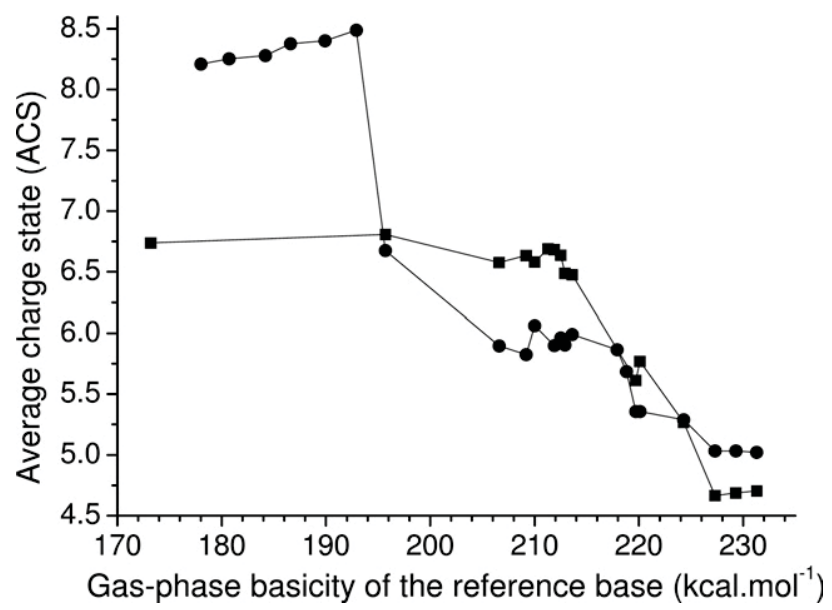

Figure 2. Average charge state (ACS) versus gas-phase basicity of the volatile references bases for lysozyme in denaturing [dashdot-dash (-)-)] and nondenaturing [dash-filled square-dash (-口-)] buffers.
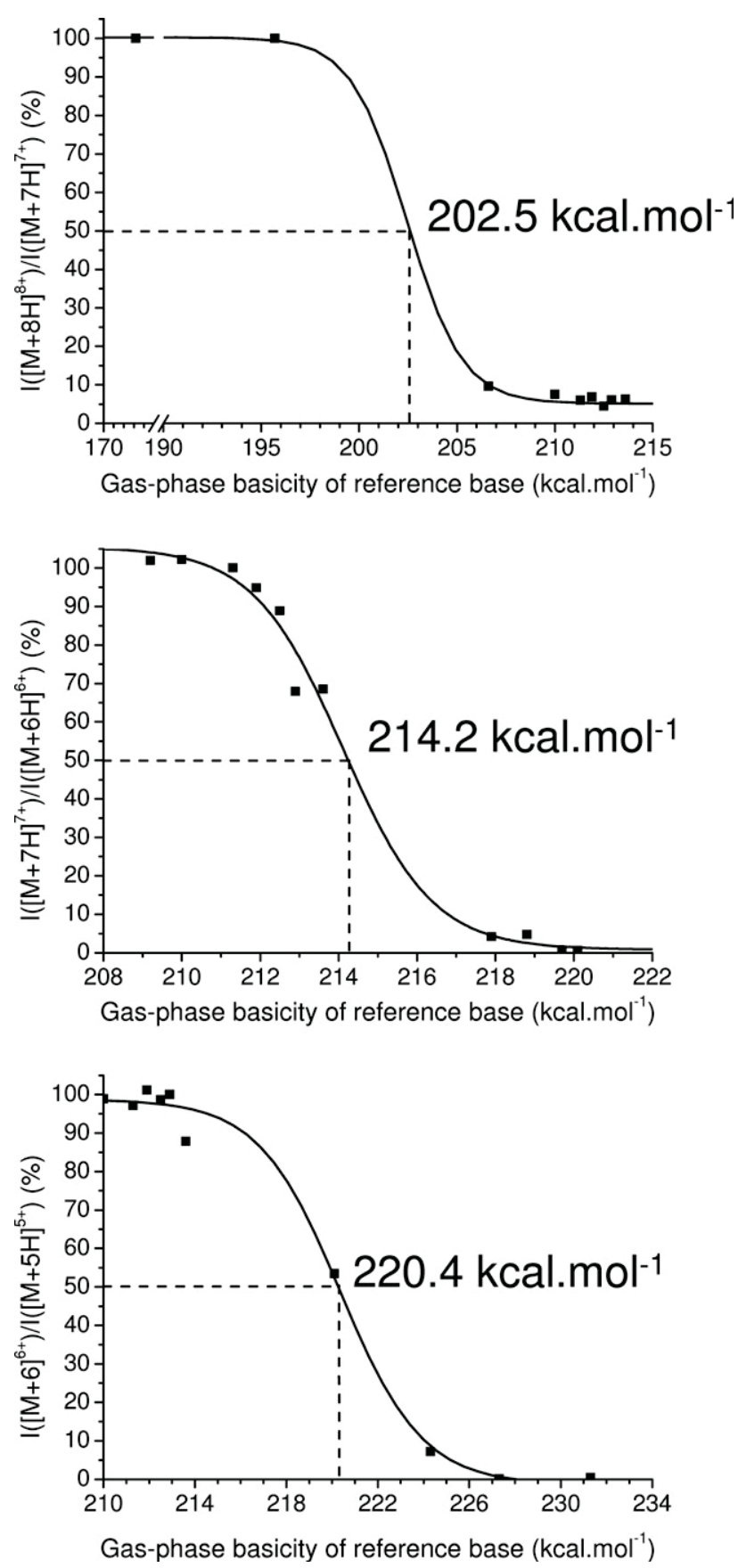

Figure 3. Relative intensity of two adjacent charge states for lysozyme in a nondenaturing buffer versus gas-phase basicity of the volatile references bases.

the $\mathrm{GB}_{\text {app }}$ values are given when a sudden break is observed for the deprotonation rate. Unfortunately, this break point is not so clear for multiply charged protein ions due to a gradual transition between endergonic and exergonic protonation reaction. Different experimental criteria can be used [15, 18, 32-34] but the best agreement between experimental and calculated values for the dication 1,7-diaminoheptane was obtained for a deprotonation efficiency of 50\% [19]. Previous results obtained in our laboratory confirmed a very good 
Table 1. Values of $\mathrm{GB}_{\mathrm{app}}$ in $\mathrm{kcal} \cdot \mathrm{mol}^{-1}$ obtained by deprotonation reaction of intact lysozyme in denaturing and nondenaturing buffers, disulfide-reduced lysozyme in a denaturing buffer, ribonuclease $\mathrm{A}$, and $\alpha$-lactalbumin in a nondenaturing buffer

\begin{tabular}{|c|c|c|c|c|c|}
\hline Charge state & $\begin{array}{c}\text { Intact lysozyme in } \\
\text { nondenaturing } \\
\text { buffer }\end{array}$ & $\begin{array}{l}\text { Intact lysozyme in } \\
\text { denaturing buffer }\end{array}$ & $\begin{array}{l}\text { Disulfide-reduced } \\
\text { lysozyme in } \\
\text { denaturing buffer }\end{array}$ & $\begin{array}{c}\text { Ribonuclease } A \text { in } \\
\text { nondenaturing } \\
\text { buffer }\end{array}$ & $\begin{array}{c}\alpha \text {-Lactalbumin in } \\
\text { nondenaturing } \\
\text { buffer }\end{array}$ \\
\hline 4 & $>231.3$ & $>231.3$ & $>231.3$ & $>231.3$ & $>231.3$ \\
\hline 5 & $>231.3$ & $>231.3$ & $>231.3$ & 225.8 & $>231.3$ \\
\hline 6 & 220.4 & 219.2 & 225.1 & 216.7 & 225.8 \\
\hline 7 & 214.2 & 202.5 & 217.5 & 214.2 & 220.4 \\
\hline 8 & 202.5 & 195.7 & 202.5 & 202.5 & $<195.7$ \\
\hline 9 & $<195.7$ & $<195.7$ & $<195.7$ & & \\
\hline 10 & & $<195.7$ & $<195.7$ & & \\
\hline 11 & & $<195.7$ & $<195.7$ & & \\
\hline 12 & & & $<195.7$ & & \\
\hline 13 & & & $<195.7$ & & \\
\hline
\end{tabular}

agreement between $\mathrm{GB}_{\mathrm{app}} \mathrm{s}$ measured by the kinetic method and ESSI-MS when an deprotonation efficiency of $50 \%$ was used [27]. Thus we decided to use this criterion to evaluate the $\mathrm{GB}_{\mathrm{app}}$ s of the multiply charged proteins (Table 1).

In denaturing buffer, deprotonation agents which are less basic than ammonia, such as alcohols, did not lead to a change in the charge-state distribution (Figure 2) for the disulfide-intact lysozyme. Using ammonia, the charge states 11,10, and 9 disappeared implying that the $\mathrm{GB}_{\mathrm{app}} \mathrm{s}$ of these charge states are below or close to the GB of ammonia and the charge state distribution resembled that obtained in nondenaturing buffer (Figure $1 b$ ). The $\mathrm{GB}_{\text {app }}$ values obtained for charge states $n 8$ to 6 are different from those obtained for the same charge state of the disulfide-intact lysozyme in the nondenaturing buffer (Table 1). Surprisingly, these $\mathrm{GB}_{\text {app }}$ values are lower than those obtained in nondenaturing condition, meaning that the disulfide-intact lysozyme is still partially folded in the gas phase after being spayed from a denaturing buffer. In the case of lysozyme, the changes in charge state distribution in this system are mediated by the four covalent disulfide linkages between the 6-127, 30-115, 64-80, and 76-94 cystine residues, therefore, the corresponding protonation sites and conformations are not available for disulfide-intact lysozyme. Reduced lysozyme in denaturing buffer was also studied. As expected more basic residues are accessible for protonation in this case leading to higher charge states and higher $\mathrm{GB}_{\text {app }}$ values than those of disulfide-intact lysozyme (Table 1). These observations provide evidence that the conformation of the protein depends on the buffer used and on the presence of disulfide bridges, which can prevent unfolding of the protein ions in the gas phase as shown by ion mobility determinations [35], leading to different ionization sites or different conformations around the same protonated sites.

To prove that the $\mathrm{GB}_{\text {app }}$ values measured by ESSI are not only dependent on the protein conformation but also on the amino acid composition, ribonuclease $\mathrm{A}$ (MW $=13,690 \mathrm{Da}, 4$ arginines, 4 histidines, 10 lysines) and $\alpha$-lactalbumin $(\mathrm{MW}=14,186 \mathrm{Da}, 1$ arginine, 3 histidines, 12 lysines) were also studied. According to the crystal structures, these two proteins have molecular masses and surface areas close to those of disulfideintact lysozyme $(\mathrm{MW}=14,313 \mathrm{Da}, 11$ arginines, 1 histidine, 6 lysines), but totally different amino acid compositions. Moreover, $\alpha$-lactalbumin and lysozyme showed similar cross-sections for all the charge states in the gas phase, measured by ion mobility experiments [36]. The $\mathrm{GB}_{\text {app }}$ values for different charge states of the three proteins are reported in Table 1. For charge state 6 , the $\mathrm{GB}_{\text {app }} \mathrm{s}$ measured for intact lysozyme, ribonuclease $\mathrm{A}$ and $\alpha$-lactalbumin are completely different, whereas the differences are less pronounced for the other charge states. It is thus clear that the experimental $\mathrm{GB}_{\text {app }}$ values depend on the amino acid composition and not on the size of the protein.

An important question is whether or not the vapor pressure of the reagent base can influence the data obtained with our set up. The vapor pressures of the bases used in our experiments differ by an estimated 2 orders of magnitude. A few simple considerations show, however, that the large differences in vapor pressure are not a problem. First, propylamine and allylamine have close vapor pressures, $\mathrm{P}_{\mathrm{v}}$, around 0.3 atm. The extent of deprotonation was always higher for propylamine than for allylamine. The reactivity clearly follows the GB order (211.3 and $209.2 \mathrm{kcal} \cdot \mathrm{mol}^{-1}$, respectively). Ethylamine has a $P_{v}$ around $1.2 \mathrm{~atm}$, four times higher than the one of propylamine or allylamine, but a GB $\left(210.0 \mathrm{kcal} \cdot \mathrm{mol}^{-1}\right)$ between propylamine or allylamine. In all cases, the extent of the deprotonation using ethylamine was higher than with allylamine and lower than with propylamine, i.e., it is the GB that dictates the reactivity, not the vapor pressure. Conversely, methylamine $\left(\mathrm{P}_{\mathrm{v}} \sim 2.9 \mathrm{~atm}\right.$ and GB $\left.206.6 \mathrm{kcal} \cdot \mathrm{mol}^{-1}\right)$ showed a lower extent of deprotonation compared to allylamine, ethylamine, or propylamine. These observations clearly demonstrate that the different vapor pressure values for the reference bases have no effect on our GB measurements.

Given in Table 2 are predicted disulfide-intact lysozyme $\mathrm{GB}_{\mathrm{app}} \mathrm{s}$ taken from literature: from the group of Kebarle [24] and the experimental data obtained by this 


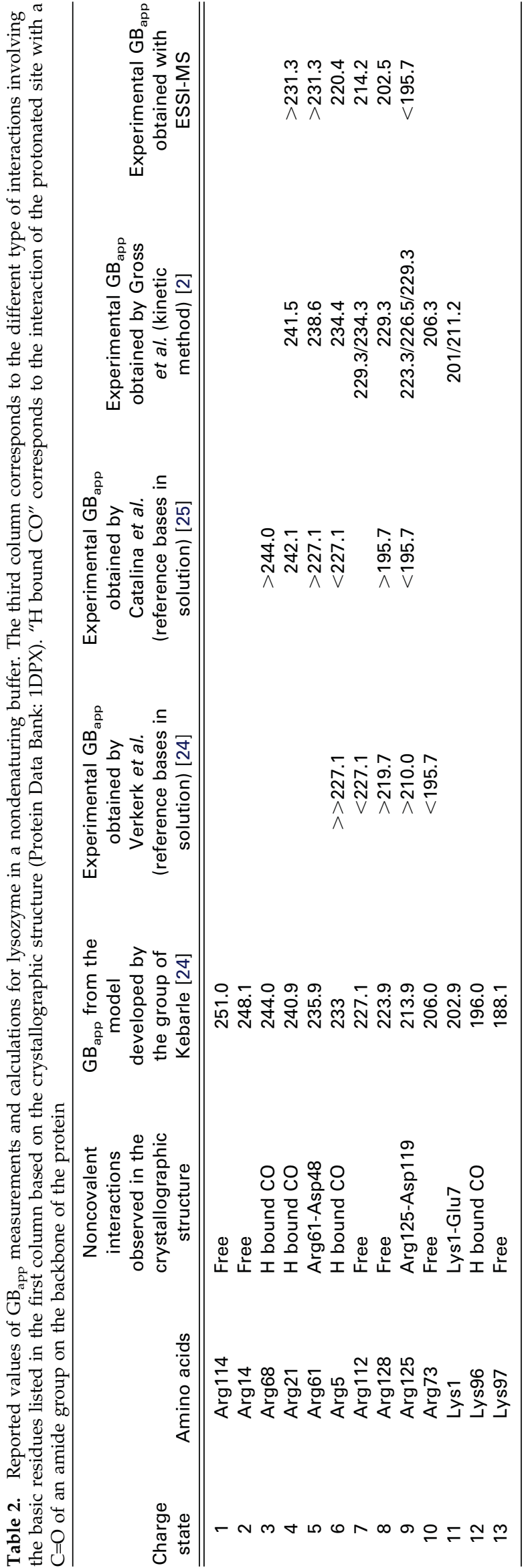

group using a different nondenaturing buffer, from Gross et al. using FT-ICR [2] and Catalina et al. using different additives in the solution, in particular compounds with very strong GB, the so-called "proton sponges" [25]. The results obtained by Gross et al. fit the model developed by Kebarle quite well, with a deviation of less than $10 \mathrm{kcal} \cdot \mathrm{mol}^{-1}$ for all the values, while the values reported by Catalina et al. are only in general agreement with the predicted $\mathrm{GB}_{\text {app }}$ due to wide spacing between reference bases. Our results obtained in nondenaturing buffer for the disulfide-intact lysozyme seem to be in poor agreement with the predicted values.

First, it must be noted that the maximum charge states for the disulfide-intact lysozyme observed by Gross et al. and Catalina et al. are 11 and 12, respectively, surprisingly high for folded protein ions in the gas phase. In fact, de la Mora has provided clear evidence that the maximum charge $Z_{\max }$ carried by a folded protein ion, approximated as a spherical structure, is close to the Rayleigh limit of solvent droplets of the same size as the protein, and can be easily calculated by the formula:

$$
Z_{\max }=0.0778 \times \sqrt{M W}
$$

where MW is the molecular weight of the protein [29]. Excellent agreement between the predicted and observed maximum charge state of 79 proteins and noncovalent complexes was demonstrated by Nesatyy and Suter [37]. Table 3 reports the $Z_{\max }$ values calculated by this model and the experimental values obtained with ESSI on nine proteins in a nondenaturing buffer. Thus, the maximum charge state accessible for the folded lysozyme in the gas phase should be only 10 . This value is in good agreement with the values obtained by Verkerk et al. with nanospray [24] and with our ESSI experiments using nondenaturing conditions, but in poor agreement with the values obtained in water by Catalina et al. [25] and Gross et al. [2]. Partial unfolding or different configuration in the gas phase are thought to be the reason, which is supported by the assignment of at least two different $\mathrm{GB}_{\text {app }}$ values for lower charge states of the disulfide-intact lysozyme sprayed in water. In this case the results reported in water must be regarded with caution.

The model developed by the group of Kebarle is based on corrections of the intrinsic GB of each basic site (arginine, lysine, and histidine) by taking into account stabilization of the charge by the protein environment [22]. The first step is to calculate the electrostatic repulsions between the protonated basic residues using the X-ray or NMR structure. Only basic sites on the surface of the protein which are accessible to the solvent are taken into account. The deprotonation agent is then positioned at the center of charge of all protonated residues and moved away by $6.5 \AA$. This new position is assumed to be close to the position of the transition-state for proton transfer between the protonated protein and the external neutral base. By correcting 
Table 3. Calculated and observed molecular weight and maximal charge state $\left(\mathrm{Z}_{\max }\right.$ [37]) for different proteins sprayed by ESSI from a nondenaturing buffer

\begin{tabular}{lcccc}
\hline \multicolumn{1}{c}{ Protein } & Theoretical molecular weight & $\begin{array}{c}\text { Experimental } \\
\text { molecular weight }\end{array}$ & $Z_{\text {max }}$ calculated & $Z_{\text {max }}$ observed \\
\hline \hline Ubiquitin & 8565 & 8565 & 7 & 6 \\
Cytochrome $c$ & 12373 (protein + heme + iron) & 12371 & 8 & 8 \\
Lysozyme & 14313 (4 disulfide bridges) & 14310 & 10 & $9-10$ \\
& 14305 (reduced form) & 14302 & 10 & 10 \\
-Lactalbumin & 14186 & 14185 & 10 & 10 \\
Ribonuclase A & 13690 & 13685 & 9 & 10 \\
Myoglobin & 16946 & 16950 & 9 & 10 \\
Hemoglobin $\alpha$ & 15053 & 15960 & 13 & 12 \\
Hemoglobin $\beta$ & 15954 & 29160 & 12 & \\
Human carbonic anhydrase II & 26114 & & & 10 \\
\hline
\end{tabular}

the intrinsic GB of the protonated sites with electrostatic repulsion energies and polarization effects, the $\mathrm{GB}_{\text {app }}$ of all the basic residues can be evaluated. This model is based on the assumption that all acidic sites are neutral in the gas phase. It is known that most of the basic sites are protonated and most of the acidic sites are deprotonated in solution at $\mathrm{pH} \sim 7$. It is well known that residues with opposite charges close in the protein structure can form salt bridges and thereby participate in the stabilization of a folded tertiary structure. Deprotonated acidic sites can also be protected by interactions with some polar groups of the protein. Grandori and coworkers extended these tenets to develop the conformation dependent neutralization $(\mathrm{CDN})$ theory $[38,39]$. They proposed that the charge state of folded proteins in the gas phase corresponds to the difference between the number of basic and the acidic sites. Nesatyy and Suter tested this theory on 79 proteins and noncovalent complexes but found a poor agreement between this model and the experimental data [37]. Nevertheless, X-ray or NMR structures were not used to determine the exact number of salt bridges and their localization on the surface of the protein. This effort was later on made by Prakash and Mazumdar [40]. They showed that salt bridges and hydrogen bonds must be taken into account to determine the maximum charge state of protein in the positive and negative ion modes. Moreover, they showed that the number of potassium adducts in the positive ion mode, when using potassium acetate in solution (K:protein $=1: 10)$ is equal to the main charge state of the protein ion in the negative ion mode. With these simple experiments, they proved that only five of a total of 15 acidic sites of cytochrome $c$ can be cationized by potassium and only seven of 24 for myoglobin. They concluded that most acidic sites are protected against cationization by hydrogen bonds or salt bridges. Salt bridges between ionized basic and acidic sites can surely survive to soft ionization, such as ESI or ESSI. In contrast, when using sodium salts in the solution molar ratio of 1 to 1000 (Na:protein), hydrogen bounds and salt bridges are totally destroyed, leading to a maximum charge state corresponding to the sum of the number of acidic sites and the number of charges [3], as predicted by the simple CDN model.
We propose to take into account all possible salt bridges between ionized basic and acidic sites close on the protein surface to correct the model developed by the group of Kebarle. Three interesting salt bridges between arginine 61/aspartic acid 48, arginine 125/ aspartic acid 119, lysine 1/glutamic acid 7 (Table 2) were identified after a careful examination of the threedimensional structure of lysozyme (PDB: 1DPX). Arginine5 , Arginine 21, Arginine 68, and Lysine 96 could be also involved in hydrogen bonds with amide groups of the protein backbone. As the model of Kebarle accounts for polarization effects, we assume that the basic sites involved in hydrogen bounds with amide groups are still accessible to ionization and that the predicted $\mathrm{GB}_{\text {app }}$ values must be close to the experimental values. Table 4 shows the corrected assignment of $\mathrm{GB}_{\text {app }}$ values in comparison with our experimental data. A good agreement is obtained, especially for the extreme charge states 4, 5, 8, and 9. For charge states 6 and 7 the deviation may be explained by errors in the experimental $\left( \pm 2 \mathrm{kcal} \cdot \mathrm{mol}^{-1}\right.$ for charge state 7$)$ and predicted $\left( \pm 5 \mathrm{kcal} \cdot \mathrm{mol}^{-1}\right)$ values. Moreover, two different conformations with very similar cross sections were suspected by cross section measurements using ion mobility mass spectrometry [35].

On one hand, predicted and experimental values of $\mathrm{GB}_{\text {app }}$ obtained with ESI-FT-ICR (kinetic method [2]) or nanoESI-TOF (different buffers [24, 25]) are in good agreement for lysozyme. As discussed in the introduction, these results must be regarded with caution. On the other hand, our experiments realized with ESSI and the experiments made by Prakash and Mazumdar with ESI seem to confirm the conservation of salt bridges in the gas phase supporting the validity of a refined Kebarle's model. The types of ionization sources and mass analyzers can have influence on the $\mathrm{GB}_{\text {app }}$ measurements due to different protein ion conformations preserved in the gas phase. ESSI is a very gentle ionization technique leading to folded proteins and maintenance of noncovalent complexes in the gas phase [28, 41]. The fast droplet evaporation due to the high nebulizing gas pressure, which could "freeze" the studied system, may be at the origin of this. Our results on lysozyme in nondenaturing buffer show that salt 
Table 4. $\mathrm{GB}_{\mathrm{app}}$ values for lysozyme calculated by the group of Kebarle [24] and refined by taking into account the possible salt bridges compared to our measurements in a nondenaturing buffer

\begin{tabular}{cccc}
\hline Charge state & $\begin{array}{c}\text { Newly protonated } \\
\text { amino acids }\end{array}$ & $\begin{array}{c}\text { Corrected } \mathrm{GB}_{\text {app }} \text { from the model developed by } \\
\text { the group of Kebarle [24] }\end{array}$ & $\begin{array}{c}\text { Experimental GB } \text { app }_{\text {obtained }} \\
\text { with ESSI-MS }\end{array}$ \\
\hline \hline 1 & Arg114 & 251.0 & $>231.3$ \\
2 & Arg14 & 248.1 & $>231.3$ \\
3 & Arg68 & 244.0 & 220.4 \\
4 & Arg21 & 240.9 & 214.2 \\
5 & Arg5 & 233 & 202.5 \\
6 & Arg112 & 227.1 & $<195.7$ \\
7 & Arg128 & 223.9 & 206.0 \\
8 & Arg73 & 196.0 & 188.1 \\
\hline
\end{tabular}

bridges are conserved in the gas phase with ESSI, but destroyed when using harsher conditions.

\section{Ubiquitin}

Taking into account the possible salt bridges between arginine 74/aspartic Acid 39, lysine 27/aspartic acid 52, and lysine $63 /$ glutamic acid 64 identified on the crystallographic structure of ubiquitin (PDB: 1UBQ, human ubiquitin identical in sequence and 3D structure than bovine ubiquitin), Kebarle's model was refined and compared to the experimental values obtained with ESSI-MS in denaturing and nondenaturing buffer (Table 5). Arginine 72 is on the protein surface, fully accessible to the solvent for protonation but is missing in the analysis presented by Verkerk et al. As Arginine has an intrinsic GB of $251 \mathrm{kcal} \cdot \mathrm{mol}^{-1}, 14 \mathrm{kcal} \cdot \mathrm{mol}^{-1}$ above that of histidine or lysine, arginine 72 was added in Table 5 but without any $\mathrm{GB}_{\text {app }}$ value. Our experimental data are in very good agreement with the predicted values corrected by the presence of salt bridges.

It must be mentioned that in ammonium bicarbonate a back shift is observed when plotting the intensity ratio between the charge state 5 and 4 against the GB of the volatile deprotonation agents (Figure 4). Up to ammonia, this ratio is constant and equal to about 2.6, but for GBs of the volatile base between $206.6 \mathrm{kcal} \cdot \mathrm{mol}^{-1}$ (methylamine) and $215.0 \mathrm{kcal} \cdot \mathrm{mol}^{-1}$ (cyclohexylamine) this ratio increases. An increase of the signal-to-noise ratio was also observed. A sudden break is obtained for diethylamine $\left(219.7 \mathrm{kcal} \cdot \mathrm{mol}^{-1}\right)$ due to the complete disappearance of the charge state 5 from the spectrum.
In pure water, the sensitivity was low because only $\mathrm{H}_{3} \mathrm{O}^{+}$ions, produced by autoprotolysis of water, can act as proton donors. Nevertheless, the back-shift for aqueous solution was observed from ammonia through diethylamine. This phenomenon could be rationalized by the fact that the observed charge state distribution depends on the number of accessible protons and on Coulombic repulsions. The group of Kebarle gave some evidence that, when using nondenaturing buffer, the first step after the droplet fission is the neutralization of the charged residues on the protein surface by ion pairing [3]. Then the charging of the protein by remaining $\mathrm{NH}_{4}{ }^{+} / \mathrm{H}_{3} \mathrm{O}^{+}$ions, when using ammonium salts or water as nondenaturing buffer, respectively, occurs via transfer proton reactions controlled by the GB. Finally, during the so called clean-up stages, small aggregates, formed by the multiply charged protein and some remaining $\mathrm{NH}_{4}{ }^{+} / \mathrm{H}_{3} \mathrm{O}^{+}$ions and some neutrals (mainly ammonia and ammonium salt ion pairs/water) are dissociated. It was already shown that the energy supplied to achieve desolvation may also lead to charge loss [42], which could explain the back-shift phenomenon.

\section{Cytochrome c}

The case of cytochrome $c$ is much more complicated. The $\mathrm{GB}_{\text {app }}$ values for different charge states of cytochrome $c$ in denaturing and nondenaturing buffer were experimentally measured (Table 6). It must be noted that the group of Kebarle also had some difficulties to obtain useable results for cytochrome $c$ due to denaturation processes occurring in solution when electro-

Table 5. $\mathrm{GB}_{\mathrm{app}}$ values for ubiquitin calculated by the group of Kebarle [24] and refined by taking into account the possible salt bridges compared to our measurements in the nondenaturing buffer

\begin{tabular}{cccc}
\hline Charge state & $\begin{array}{c}\text { Newly protonated } \\
\text { amino acids }\end{array}$ & $\begin{array}{c}\text { Corrected } \mathrm{GB}_{\text {app }} \text { from the model developed by } \\
\text { the group of Kebarle [24] }\end{array}$ & $\begin{array}{c}\text { Experimental GB } \text { app }_{\text {obtained }} \\
\text { with ESSI-MS }\end{array}$ \\
\hline \hline 1 & Arg54 & 251.0 & $>231.3$ \\
2 & Arg72 & - & 225.8 \\
3 & Arg42 & 241 & 216.5 \\
4 & Lys33 & 223 & $<195.7$ \\
5 & Lys6 & 211 & - \\
6
\end{tabular}




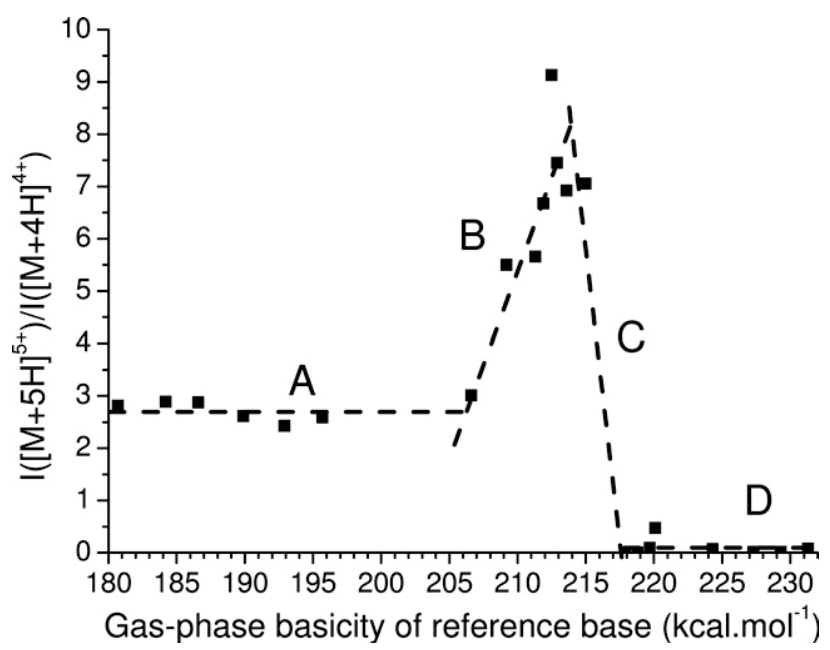

Figure 4. Relative intensity of the charge states 4 and 5 for ubiquitin in a nondenaturing buffer versus gas-phase basicity of the volatile references bases.

spraying over long time [24]. In denaturing buffer, the charge states $n 9$ to 17 have exactly the same $\mathrm{GB}_{\text {app }}$, close to that of ammonia $\left(195.7 \mathrm{kcal} \cdot \mathrm{mol}^{-1}\right)$, while the $\mathrm{GB}_{\text {app }}$ of charge state 8 is close to that of methylamine. For the charge state 7 , two $\mathrm{GB}_{\text {app }}$ values can be assigned due to two different transitions (Figure 5), meaning that two distinct conformations must coexist in the gas phase. For the charge state 6, only one $\mathrm{GB}_{\text {app }}$ value was determined, although three distinct conformations have been observed for charge state 7 of cytochrome $c$ sprayed from denaturing solution using ion mobility measurements [43]. In the nondenaturing buffer, only one $\mathrm{GB}_{\text {app }}$ was attributed for each charge state. These values are close to those obtained in denaturing buffer. Cytochrome $c$ ions are quite sensitive to the mass spectrometric conditions. The group of Grandori proved that cytochrome $c$ is prone to denaturation when increasing the curtain gas flow or the $\mathrm{pH}$ [44]. Due to the supersonic nebulizing gas used for ESSI measurements, partial denaturation is possible and a

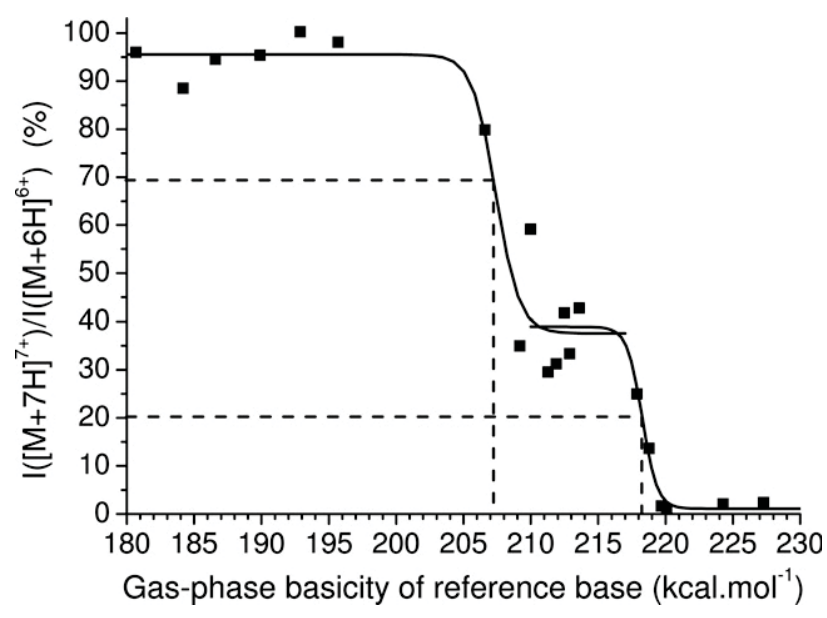

Figure 5. Relative intensity of the charge states 6 and 7 for cytochrome $c$ in a denaturing buffer versus gas-phase basicity of the volatile references bases.

considerable number of salts bridges on the protein surface could be destroyed. Taking this into account, and considering the fact that the iron in the heme can contribute one positive charge, a good correlation between the estimated and experimental $\mathrm{GB}_{\text {app }}$ values was demonstrated.

\section{Human Carbonic Anhydrase II and Creatine Kinase}

Denaturing buffer produces fully unfolded but also partially folded protein ions. The ion population distribution depends strongly on the charge states and many different ion conformations were observed by ion mobility spectrometry for lysozyme [35], ubiquitin [45] and cytochrome $c$ [43] in denaturing buffer. If different conformations with different $\mathrm{GB}_{\mathrm{app}}$ coexist in the gas phase, bi(/multi)modal charge-state distributions should be observed after proton transfer, at least under certain experimental conditions, reflecting the different

Table 6. Experimental values of $\mathrm{GB}_{\mathrm{app}} \mathrm{s}$ for cytochrome $\mathrm{c}$ in denaturing and nondenaturing buffers obtained by ESSI-MS compared to the calculations made by the group of Kebarle [24]

\begin{tabular}{|c|c|c|c|c|}
\hline $\begin{array}{c}\text { Charge } \\
\text { state }\end{array}$ & Amino acids & $\begin{array}{l}\text { Corrected } \mathrm{GB}_{\mathrm{app}} \text { from the } \\
\text { model developed by the } \\
\text { group of Kebarle [24] }\end{array}$ & $\begin{array}{c}\text { Experimental } \mathrm{GB}_{\mathrm{app}} \text { obtained with } \\
\text { ESSI-MS for cytochrome } \mathrm{c} \text { in non- } \\
\text { denaturing buffer }\end{array}$ & $\begin{array}{c}\text { Experimental } \mathrm{GB}_{\mathrm{app}} \text { obtained with } \\
\text { ESSI-MS for cytochrome } \mathrm{c} \text { in } \\
\text { denaturing buffer }\end{array}$ \\
\hline 1 & Heme & & & \\
\hline 2 & Arg38 & 251 & & \\
\hline 3 & Arg91 & 247 & & \\
\hline 4 & Lys7 & 229 & $>231.3$ & $>231.3$ \\
\hline 5 & Lys55 & 225 & $>231.3$ & $>231.3$ \\
\hline 6 & Lys13 & 221 & 223.5 & 225.8 \\
\hline 7 & Lys72 & 216 & 218.3 & $207.4 / 218.3$ \\
\hline 8 & His26 & 212 & 206.6 & 206.6 \\
\hline 9 & Lys53 & 209 & & $<195.7$ \\
\hline 10 & Lys27 & 203 & & $<195.7$ \\
\hline 11 & Lys99 & 198 & & $<195.7$ \\
\hline 12 & Lys22 & 195 & & $<195.7$ \\
\hline 13 & & & & $<195.7$ \\
\hline
\end{tabular}



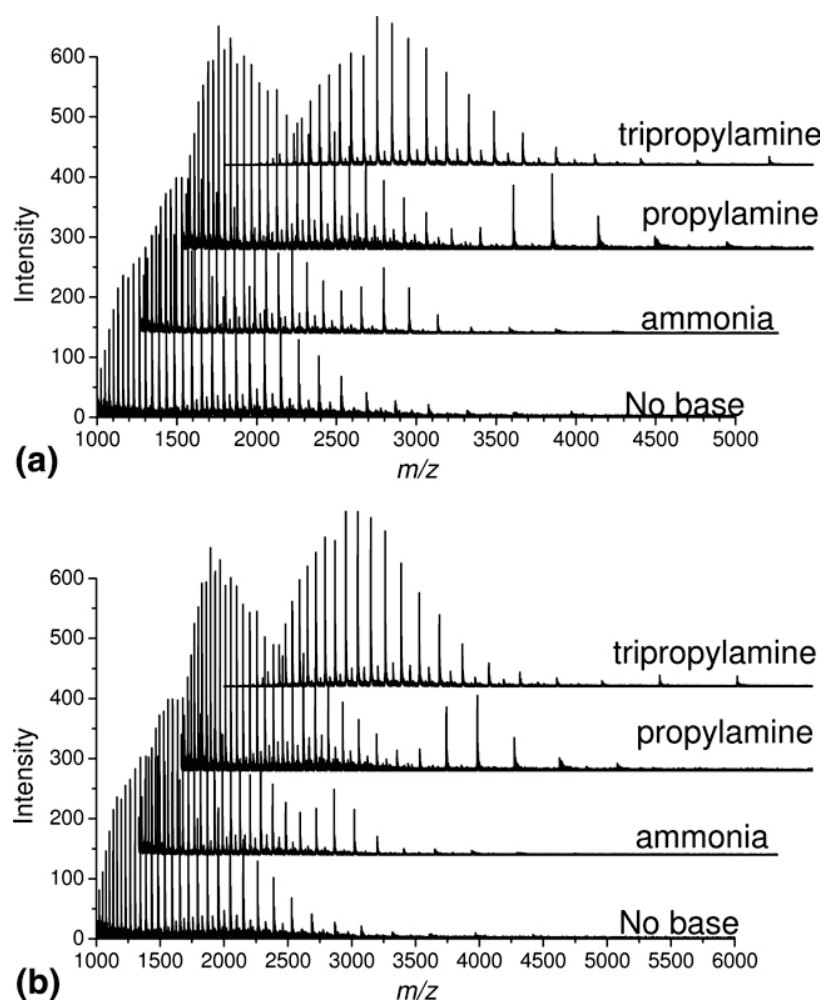

Figure 6. Representative mass spectra obtained by reacting human carbonic anhydrase II (a) or creatine kinase (b) ions sprayed by ESSI from a denaturing buffer and different neutral volatile reference bases.

reaction rates for equally charged ions. Interestingly, only one $\mathrm{GB}_{\text {app }}$ value was attributed with ESSI-MS, except for the charge state 7 of cytochrome $c$. This could be explained by the fact that ESSI is capable of "freezing" the system studied and of retaining only one of the conformations for these three proteins. In the case of human carbonic anhydrase II and creatine kinase in denaturing buffer, bimodal distributions were observed when increasing the GB of the volatile reference base (Figure 6). A first ion population $(\mathrm{m} / \mathrm{z} 1000-1800$ with no base) is quite insensitive to deprotonation while a second ion population $(\mathrm{m} / \mathrm{z}$ 1800-3000 with no base) behaved like a partially folded protein in the gas phase. For the first time, as far as we know, coexistence of two or more distinct gas-phase ion conformations is demonstrated for these proteins. To confirm our analysis, ion mobility experiments should be carried out in the future.

\section{A Unified Model for ESSI Ion Production}

While the small ions are likely to be produced by the ion evaporation model (IEM) [46], large macro ions, such as nondenatured proteins seem to be produced by the charge residue model (CRM) $[29,47]$. The IEM model developed by Iribarne and Thomson states that solvated ions are emitted directly from charged droplets after the radii of the droplets decrease to a suitable size.
At an intermediate stage of the droplet fission, the charge density on the droplet surface is lower than the Rayleigh limit but sufficient to eject a charged analyte, solvated by few solvent molecules [46]. On the other hand, Dole and coworkers proposed a succession of Coulomb fissions leading to a naked ion retaining all the charge of the initial droplet [48, 49]. Taking into accounts our observations and the pioneering research of the groups of de la Mora, Kebarle, Cole [47] and Mazumdar, we can propose a unified model for the electrosonic spray ionization process for folded and stable proteins in the gas phase, which is based on the accepted ion formation model for ESI:

1. "Charging by proton transfer reaction in the gas phase". The group of Kebarle tried to understand the chemistry of the charging of globular proteins in nondenaturing buffers, such as ammonium acetate [3]. In the first steps, ions pairs between $\mathrm{NH}_{4}{ }^{+}$ions and ionized acidic groups and acetate/carbonate ions and ionized basic groups of the protein are formed in solution. During the desolvation process, these ion pairs are gradually destroyed leading to the formation of neutral acidic and basic sites. When the solvent is reduced by evaporation to a (few) mono-layer(s) over the protein, gas-phase conditions prevail. Our experiments confirm that the charging of the protein is controlled by GB. Excess $\mathrm{NH}_{4}{ }^{+}$on the surface of the droplets will provide charges to the protein by protonation reactions, which only occurs when the $\mathrm{GB}_{\text {app }}$ of the basic site of the protein is higher than the GB of $\mathrm{NH}_{4}{ }^{+}$.

2. "The maximum charge state depends on the volume of the folded protein". De la Mora has provided good evidence that the folded protein ions are produced by the CRM model [29]. The idea was to consider that after some fission events the volume of the droplet is close to the volume of the folded protein and that the droplet will transfer all the charge to the mostly neutral protein. Beauchamp and coworkers provided evidence that the droplets remain close to the Rayleigh limit after fissions [50]. Thus, the maximum protein charge will be equal to the charge $Z_{\max }$ of a droplet at the Rayleigh limit, with the same size of the protein. $Z_{\max }$ can be calculated with the Rayleigh equation and the radius of the folded protein, estimated by X-ray or NMR data. This model was developed in the case of ESI. Our results for ESSI are fully consistent with this model, meaning that a faster evaporation should not disturb too much the last step of the fission process.

3. "The charge state distribution of a folded protein is determined by both $\mathrm{GB}_{\mathrm{app}}$, the maximal charge $\mathrm{Z}_{\max }$, and the Coulombic repulsions". If the number of basic sites which can hold a proton in the gas phase is lower than $Z_{\max }$, the charge state distribution will be determined by the $\mathrm{GB}_{\text {app }}$ values. Otherwise, the charge distribution will be determined by 
the Rayleigh limit. The back-shift observed for ubiquitin and cytochrome $c$ with some reference volatile bases demonstrated the important role that Coulombic repulsions play in the ionization process. A higher signal-to-noise ratio and a shift of the charge state distribution to higher $m / z$ values can be observed when a volatile reference base with a high steric volume is used.

4. "Salt bridges can be maintained in the gas phase". As showed by Prakash and Mazumdar [40] and by our results on lysozyme and ubiquitin, the salt bridges between deprotonated acidic sites and protonated basic sites close on the protein surface can be maintained in the gas phase if the ionization process is very soft or if the folded conformation of the protein is sufficiently stable in the gas phase. This point is quite surprising regarding to a thermodynamic point of view. In fact, deprotonated carboxylic groups are more basic than protonated basic groups. When adding a high amount of sodium salts, these salt bridges are destroyed leading to cationization of all the acidic sites and some basic sites [3]. Thus, the $\mathrm{GB}_{\mathrm{app}}$ values are directly linked to the ion gas-phase conformation, which are expected to be close to the crystal structures for well structured proteins as a first approximation. In the case of ESSI, the fast droplet evaporation due to the high nebulizing gas pressure could "freeze" the system in one specific conformation.

The question of the charge state distribution for proteins dissolved in denaturing buffer remains difficult and no general rules can be formulated. In fact, ion mobility [40, 43, 45], H/D exchange [17] measurements, as well as our results showed that several ion conformations can coexist in the gas phase when proteins are sprayed from denaturing solution. Structural interconversions can occur depending on the time scale of the experiments [16, 51]. Coupling ESSI-MS with ion mobility mass spectrometry could be a very interesting tool to further continue these studies.

\section{Conclusions}

ESSI-MS offers the unique possibility to measure the $\mathrm{GB}_{\mathrm{app}}$ values of proteins at atmospheric pressure with good sensitivity (for concentrations less than $10 \mu \mathrm{M}$ in denaturing or nondenaturing buffer), good accuracy, in a short time (less than 30 min to screen up to 23 volatile bases) and in denaturing or nondenaturing buffers. We proved that the electrostatic model developed by the group of Kebarle and the Charge Residue Model are valid for ESSI when taking into account the salt bridges on the surface of the protein.

As demonstrated by the group of Cooks, noncovalent complexes can also be observed by ESSI [41]. Our group has very recently shown that ESSI produces noncovalent complexes in the gas phase more easily than ESI and nanospray [30]. The difference of time scale of the desolvation could be an explanation of the increase of stability observed with ESSI. The fast droplet evaporation during ESSI process, due to the high nebulizing gas pressure, seems to be able to "freeze" the studied system in one specific conformation. Further study is under progress in our laboratory to better understand the links between the ionization mechanism, the different conformation in the gas phase and the experimental data obtained on noncovalent complexes.

\section{Acknowledgments}

The authors thank Novartis Institutes for BioMedical Research for financial support.

\section{References}

1. Fenn, J. B.; Mann, M.; Meng, C. K.; Wong, S. F.; Whitehouse, C. M. Electrospray Ionization for Mass Spectrometry of Large Biomolecules. Science 1989, 246, 64-71.

2. Gross, D. S.; Schnier, P. D.; Rodriguez-Cruz, S. E.; Fagerquist, C. K.; Williams, E. R. Conformations and Folding of Lysozyme Ions in Vacuo. Proc. Natl. Acad. Sci. U.S.A. 1996, 93, 3143-3148.

3. Verkerk, U. H.; Kebarle, P. J. Ion-Ion and Ion-Molecule Reactions at the Surface of Proteins Produced by Nanospray. Information on the Number of Acidic Residues and Control of the Number of Ionized Acidic and Basic Residues. J. Am. Soc. Mass Spectrom. 2005, 16, 1325-1341.

4. Williams, E. R. Proton Transfer Reactivity of Large Multiply Charged Ions. J. Mass Spectrom. 1996, 31, 831-842.

5. Kaltashov, I. A.; Fabris, D.; Fenselau, C. C. Assessment of Gas-Phase Basicities of Protonated Peptides by the Kinetic Method. J. Phys. Chem. 1995, 99, 10046-10051.

6. Kaltashov, I. A.; Fenselau, C. C. A Direct Comparison of "First" and "Second" Gas-Phase Basicities of the Octapeptide RPPGFSPF. J. Am. Chem. Soc. 1995, 117, 9906-9910.

7. Gorman, G. S.; Speir, J. P.; Turner, C. A.; Jonathan Amster, I. Proton Affinities of the 20 Common $\alpha$-Amino Acids. J. Am. Chem. Soc. 1992, 114 3986-3988.

8. Gorman, G. S.; Amster, I. J. Kinetic and Thermodynamic Considerations of the Bracketing Method: Entropy-Driven Proton-Transfer Reactions in a Fourier Transform Mass Spectrometer. Org. Mass Spectrom. 1993, 28, 1602-1607.

9. Arshadi, M.; Yamdagni, R.; Kebarle, P. J. Hydration of the Halide Negative Ions in the Gas Phase. J. Phys. Chem. 1970, 74, 1475-1482.

10. Cunningham, A. J.; Payzant, J. D.; Kebarle, P. J. A Kinetic Study of the Proton Hydrate $\mathrm{H}^{+}\left(\mathrm{H}_{2} \mathrm{O}\right)_{n}$ Equilibria in the Gas Phase. J. Am. Chem. Soc. 1972, 94, 7627-7632.

11. Winger, B. E.; Light-Wahl, K. J.; Smith, R. D. Gas-Phase Proton Transfer Reactions Involving Multiply Charged Cytochrome $c$ Ions and Water Under Thermal Conditions. J. Am. Soc. Mass Spectrom. 1992, 3, 624-630.

12. Ogorzalek-Loo, R. R.; Smith, R. D. Investigation of the Gas-Phase Structure of Electrosprayed Proteins Using Ion-Molecule Reactions. J. Am. Soc. Mass Spectrom. 1994, 5, 207-220.

13. Cassady, C. J.; Wronka, J.; Kruppa, G. H.; Laukien, F. H. Deprotonation Reactions of Multiply Protonated Ubiquitin Ions. Rapid Commun. Mass Spectrom. 1994, 8, 394-400.

14. Cassady, C. J. Gas-Phase Reactivity and Molecular Modeling Studies on Triply Protonated Dodecapeptides that Contain Four Basic Residues. J. Am. Soc. Mass Spectrom. 1998, 9, 716-723.

15. Ewing, N. P.; Pallante, G. A.; Zhang, X.; Cassady, C. J. Gas-Phase Basicities for Ions from Bradykinin and Its Des-Arginine Analogues. J. Mass Spectrom. 2001, 36, 875-881.

16. Badman, E. R.; Myung, S.; Clemmer, D. E. Evidence for Unfolding and Refolding of Gas-Phase cytochrome $c$ Ions in a Paul Trap. J. Am. Soc. Mass Spectrom. 2005, 16, 1493-1497.

17. Horn, D. M.; Breuker, K.; Frank, A. J.; McLafferty, F. W. Kinetic Intermediates in the Folding of Gaseous Protein Ions Characterized by Electron Capture Dissociation Mass Spectrometry. J. Am. Chem. Soc. 2001, 123, 9792-9799.

18. Schnier, P. D.; Gross, D. S.; Williams, E. R. Electrostatic Forces and Dielectric Polarizability of Multiply Protonated Gas-Phase Cytochrome c Ions Probed by Ion/Molecule Chemistry. J. Am. Chem. Soc. 1995, 117, 6747-6757.

19. Gronert, S. An ab Initio Study of Proton Transfers from Gas-Phase Dications: Complications in Kinetic Methods for Determining Acidities. J. Am. Chem. Soc. 1996, 118, 3525-3526.

20. Gronert, S. Determining the Gas-Phase Properties and Reactivities of Multiply Charged Ions. J. Mass Spectrom. 1999, 34, 787-796. 
21. Gronert, S. Coulomb Repulsion in Multiply Charged Ions: A Computational Study of the Effective Dielectric Constants of Organic Spacer Groups. Int. J. Mass Spectrom. 1999, 185, 351-357.

22. Peschke, M.; Blades, A.; Kebarle, P. Charged States of Proteins. Reactions of Doubly Protonated Alkyldiamines with $\mathrm{NH}(3)$ : Solvation or Deprotonation. Extension of Two Proton Cases to Multiply Protonated Globular Proteins Observed in the Gas Phase. J. Am. Chem. Soc. 2002, 124, 11519-11530.

23. Lemaire, D.; Marie, G.; Serani, L.; Laprévote, O. Stabilization of GasPhase Noncovalent Macromolecular Complexes in Electrospray Mass Spectrometry Using Aqueous Triethylammonium Bicarbonate Buffer. Anal. Chem. 2001, 73, 1699-1706.

24. Verkerk, U. H.; Peschke, M.; Kebarle, P. Effect of Buffer Cations and of $\mathrm{H}_{3} \mathrm{O}^{+}$on the Charge States of Native Proteins. Significance to determinations of stability constants of protein complexes. J. Mass Spectrom. 2003, 38, 618-631.

25. Catalina, M. I. Van den Heuvel, R. H. H. Van Duijn, E.; Heck, A. J. R. Decharging of Globular Proteins and Protein Complexes in Electrospray. Chem. Eur. J. 2005, 11, 960-968.

26. Takáts, Z.; Wiseman, J. M.; Gologan, B.; Cooks, R. G. Electrosonic Spray Ionization. A Gentle Technique for Generating Folded Proteins and Protein Complexes in the Gas Phase and for Studying Ion-Molecule Reactions at Atmospheric Pressure. Anal. Chem. 2004, 76, 4050-4058.

27. Touboul, D.; Jecklin, M. C.; Zenobi, R. Rapid and Precise Measurements of Gas-Phase Basicity of Peptides and Proteins at Atmospheric Pressure by Electrosonic Spray Ionization-Mass Spectrometry. J. Phys. Chem. 2007, 111, 11629-11631.

28. Rayleigh, L. On the Equilibrium of Liquid Conducting Masses Charged with Electricity. Phil. Mag. 1882, 14, 184

29. de la Mora, J. F. Electrospray Ionization of Large Multiply Charged Species Proceeds via Dole's Charged Residue Mechanism. Anal. Chim. Acta 2000, 406, 93-104.

30. Jecklin, M. C.; Touboul, D.; Bovet, C.; Wortmann, A.; Zenobi, R. Which Electrospray-Based Ionization Method Best Reflects Protein-Ligand Interactions Found in Solution? A Comparison of ESI, nanoESI, and ESSI for the Determination of Dissociation Constants with Mass Spectrometry. I. Am. Soc. Mass Spectrom. in press.

31. http://webbook.nist.gov/chemistry/.

32. Bouchoux, G.; Salpin, J. Y.; Leblanc, D. A Relationship Between the Kinetics and Thermochemistry of Proton Transfer Reactions in the Gas Phase. Int. J. Mass Spectrom. Ion Processes 1996, 153, 37-48.

33. Wu, J.; Lebrilla, C. B. Gas-Phase Basicities and Sites of Protonation of Glycine Oligomers $\left(\mathrm{GLY}_{n} ; n=1-5\right)$. J. Am. Chem. Soc. 1993, 115, 3270-3275.

34. Wu, J.; Lebrilla, C. B. Intrinsic Basicity of Oligomeric Peptides that Contain Glycine, Alanine, and Valine-The Effects of the Alkyl Side Chain on Proton Transfer Reactions. J. Am. Soc. Mass Spectrom. 1995, 6 , 91-101.

35. Valentine, S. J.; Anderson, J. G.; Ellington, A. D.; Clemmer, D. E. Disulfide-Intact and -Reduced Lysozyme in the Gas Phase: Conforma- tions and Pathways of Folding and Unfolding. J. Phys. Chem. B 1997, 101, 3891-3900.

36. Thalassinos, K.; Slade, S. E.; Jennings, K. R.; Scrivens, J. H.; Giles, K.; Wildgoose, J.; Hoyes, J.; Bateman R. H.; Bowers, M. T. Ion Mobility Mass Spectrometry of Proteins in a Modified Commercial Mass Spectrometer. Int. J. Mass Spectrom. 2004, 236, 55-63.

37. Nesatyy, V. J.; Suter, M. J. F. On the Conformation-Dependent Neutralization Theory and Charging of Individual Proteins and Their Noncovalent Complexes in the Gas Phase. J. Mass Spectrom. 2004, 39, 93-97.

38. Grandori, R. Origin of the Conformation Dependence of Protein Charge-State Distributions in Electrospray Ionization Mass Spectrometry. J. Mass Spectrom. 2003, 38, 11-15.

39. Samalikova, M.; Grandori, R. Role of Opposite Charges in Protein Electrospray Ionization Mass Spectrometry. J. Mass Spectrom. 2003, 38, 941-947.

40. Prakash, H.; Mazumdar, S. Direct Correlation of the Crystal Structure of Proteins with the Maximum Positive and Negative Charge States of Gaseous Protein Ions Produced by Electrospray Ionization. J. Am. Soc. Mass Spectrom. 2005, 16, 1409-1421.

41. Wiseman, J. M.; Takáts, Z.; Gologan, B.; Davisson, V. J.; Cooks, R. G. Direct Characterization of Enzyme-Substrate Complexes by Using Electrosonic Spray Ionization Mass Spectrometry. Angew. Chem. Int. Ed. 2005, 44, 913-916.

42. Felitsyn, N.; Peschke, M.; Kebarle, P. Origin and Number of Charges Observed on Multiply-Protonated Native Proteins Produced by ESI. Int. J. Mass Spectrom. 2002, 219, 39-62.

43. Clemmer, D. E.; Hudgins, R. R.; Jarrold, M. F. Naked Protein Conformations: Cytochrome $c$ in the Gas Phase. J. Am. Chem. Soc. 1995, 117, 10141-10142.

44. Šamalikova, M.; Matečko, I.; Müller, N.; Grandori, R. Interpreting Conformational Effects in Protein nano-ESI-MS Spectra. Anal. Bioanal. Chem. 2004, 378, 1112-1123.

45. Koeniger, S. L.; Clemmer, D. E. Resolution and Structural Transitions of Elongated States of Ubiquitin. J. Am. Soc. Mass Spectrom. 2007, 18, 322-331.

46. Iribarne, I. V.; Thomson, B. A. On the Evaporation of Small Ions from Charged Droplets. J. Chem. Phys. 1976, 64, 2287-2294.

47. Cole, R. B. Some Tenets Pertaining to Electrospray Ionization Mass Spectrometry. J. Mass Spectrom. 2000, 35, 763-772.

48. Dole, M.; Mack, L. L.; Hines, R. L.; Mobley, R. C.; Ferguson, L. D.; Alice, M. B. Molecular Beams of Macroions. J. Chem. Phys. 1968, 49, 2240-2249.

49. Mack, L. L.; Kralik, P.; Rheude, A.; Dole, M. Molecular Beams of Macroions. II. J. Chem. Phys. 1970, 52, 4977-4986.

50. Smith, J. N.; Flagan, R. C.; Beauchamp, J. L. Droplet Evaporation and Discharge Dynamics in Electrospray Ionization. J. Phys. Chem. A 2002, 106, 9957-9967.

51. Mao, D.; Babu, K. R.; Chen, Y. L.; Douglas, D. J. Conformations of Gas-Phase Lysozyme Ions Produced from Two Different Solution Conformations. Anal. Chem. 2003, 75, 1325-1330. 